Check for updates

Cite this: RSC Adv., 2019, 9, 1134

Received 5th November 2018

Accepted 25th December 2018

DOI: $10.1039 / \mathrm{c} 8 \mathrm{ra0} 0154 \mathrm{~d}$

rsc.li/rsc-advances

\section{Re-evaluation of experimental measurements for the validation of electronic band structure calculations for $\mathrm{LiFePO}_{4}$ and $\mathrm{FePO}_{4} \dagger$}

\author{
Yin Zhang, (D) abd Jose A. Alarco, (D) *a Adam S. Best, ${ }^{\text {b }}$ Graeme A. Snook, $^{c}$ \\ Peter C. Talbot ${ }^{a}$ and Jawahar Y. Nerkar ${ }^{a}$
}

Experimental measurements used to validate previous electronic band structure calculations for olivine $\mathrm{LiFePO}_{4}$ and its delithiated phase, $\mathrm{FePO}_{4}$, have been re-investigated in this study. Experimental band gaps of $\mathrm{LiFePO}_{4}$ and $\mathrm{FePO}_{4}$ have been determined to be $6.34 \mathrm{eV}$ and $3.2 \mathrm{eV}$ by electron energy loss spectroscopy (EELS) and UV-Vis-NIR diffusion reflectance spectroscopy, respectively. X-ray photoemission (XPS) and Raman spectroscopy show that the surfaces of very carefully synthesized $\mathrm{LiFePO}_{4}$ display Li-depletion, which affects optical reflectance determinations. Based on these experimental measurements, functionals for density functional theory (DFT) calculations of the electronic properties have been revisited. Overall, electronic structures of $\mathrm{LiFePO}_{4}$ and $\mathrm{FePO}_{4}$ calculated using sXLDA show the best self-consistent match to combined experimentally determined parameters. Furthermore, the open-circuit voltages of the $\mathrm{LiFePO}_{4}$ half-cell have been interpreted in terms of both Fermi levels and Gibbs free energies, which provides additional support for the electronic band structures determined by this research.

\section{Introduction}

Concerns about greenhouse gas emissions, global warming and fossil fuel depletion are driving increased vehicle electrification as well as storage of renewable energy. ${ }^{1}$ Rechargeable Li-ion batteries (LIBs) are currently the preferred choice for hybrid electric vehicles (HEVs) and electric vehicles (EVs), due to their high energy density and relatively long cycling stability., ${ }^{2,3}$ Since the original work of Goodenough et al., ${ }^{4}$ olivine $\mathrm{LiFePO}_{4}$ has been considered as one of the best cathode materials for commercial LIBs due to its high structural stability afforded by phosphate bonds. However, the high-rate performance of $\mathrm{LiFePO}_{4}$ is greatly inhibited by its poor electronic conductivity $\left(\sim 10^{-9} \mathrm{~S} \mathrm{~cm}^{-1}\right)$ and low ionic diffusivity $\left(10^{-13}\right.$ to $\left.10^{-16} \mathrm{~cm}^{2} \mathrm{~s}^{-1}\right){ }^{5}$

\footnotetext{
${ }^{a}$ Institute for Future Environments and Science and Engineering Faculty, Queensland University of Technology (QUT), Brisbane, QLD 4001, Australia. E-mail: jose. alarco@qut.edu.au

${ }^{b}$ CSIRO Manufacturing, Private Bag 10, Clayton South, Victoria 3169, Australia ${ }^{c}$ CSIRO Mineral Resources, Private Bag 10, Clayton South, Victoria 3169, Australia ${ }^{d}$ CRRC Qingdao Sifang Rolling Stock Research Institute Co., Ltd., Qingdao 266031, China

$\dagger$ Electronic supplementary information (ESI) available: Additional optical absorption spectra, band structure calculations with NM and AFM GGA and with NM hybrid functionals, PDOS of GGA and GGA+U for $\mathrm{LIMPO}_{4}$, energy schematics of LIB systems, PDOS of $\mathrm{Fe}$ in $\mathrm{LiFePO}_{4}$ and $\mathrm{FePO}_{4}$ with various functionals, UPS spectra for $\mathrm{LiFePO}_{4}$ and $\mathrm{FePO}_{4}$ and brief summary on previous band gap estimation of $\mathrm{LiFePO}_{4}$ with GGA+U. See DOI: 10.1039/c8ra09154d
}

In order to improve these properties, a range of experimental and theoretical techniques have been deployed to understand the electronic band structure and conduction mechanisms of $\mathrm{Li}_{x} \mathrm{FePO}_{4}$ phases $(0 \leq x \leq 1)$. To optimize the materials for LIBs, theoretical computational approaches can be applied to provide guidance for experimental developments.

Calculations within the framework of density functional theory (DFT) have been widely used to explain the electronic band structure, Li insertion voltage, Li diffusivity and phase stability for Li-ion intercalation materials. ${ }^{6-8}$ However, for a predictive use of DFT attempting to enhance battery properties using modified compositions and structures, it is imperative to carry out a thorough experimental validation of the theoretical approaches, checking on the overall consistency of a variety of complementary pieces of experimental information. In addition, the theoretical approaches need to be truly selfconsistent, that is, they cannot rely on parameters that may require adjustments for each structural modification.

The band gap is a major factor determining the electronic conductivity of a solid, and it is an important characteristic of battery materials. In the case of $\mathrm{LiFePO}_{4}$ and $\mathrm{FePO}_{4}$, a wide range of experimentally determined band gap values have been reported in the literature ${ }^{9-13}$ (these are summarized and compared in detail in Discussions Section 6.1). Along with the disagreement on experimental determinations, various DFT functionals and parameters have been applied in the electronic structure calculations in previous studies with varying results. 
Antiferromagnetic transitions at $52 \mathrm{~K}$ and at $125 \mathrm{~K}$ for $\mathrm{LiFePO}_{4}$ and $\mathrm{FePO}_{4}$, respectively, have been confirmed experimentally. ${ }^{14,15}$ Accordingly, antiferromagnetic (AFM) configurations have been set up in some DFT calculations,${ }^{16}$ since DFT is, in principle, a ground state theory (valid at absolute $0 \mathrm{~K}$ ). However, in terms of the battery applications, we are more interested in properties at room temperature, where the magnetic order is paramagnetic for both $\mathrm{LiFePO}_{4}$ and $\mathrm{FePO}_{4}$. As a consequence, calculations with alternative ferromagnetic (FM) and non-magnetic (NM) configurations have also been reported in previous literature. ${ }^{\mathbf{9 1 7 - 2 0}}$ By using the local density approximation (LDA) with ferromagnetic configuration, Xu et $a l .{ }^{21}$ claimed that $\mathrm{LiFePO}_{4}$ is a half metal with spin-down Fe3d states across the Fermi level, while the generalized gradient approximation (GGA) with antiferromagnetic configuration showed a band gap of $0.5 \mathrm{eV} .^{16}$

It is well established that the LDA and GGA functionals generally underestimate band gaps for semiconductors, insulators and strongly correlated systems, to the point that it is usually referred to as 'the band gap problem'.22 Because of the presence of partially filled highly localized 3d-orbitals in $\mathrm{Fe}^{2+/ 3+}$ ions, $\mathrm{LiFePO}_{4}$ and $\mathrm{FePO}_{4}$ are grouped with the strongly correlated systems, for which the band gap would be severely underestimated using LDA and GGA. ${ }^{23-25}$ Therefore, DFT (LDA or GGA) with Hubbard U corrections (DFT+U), as a low-cost correction method, has become the most popular functional used in the electronic structure calculation of $\mathrm{LiFePO}_{4}$ and $\mathrm{FePO}_{4}$. Hubbard $\mathrm{U}$ is a Coulomb parameter, which introduces a repulsion between the localized electrons in $\mathrm{d}$ or $\mathrm{f}$ orbitals, which can cause a split of these orbitals and thereby can open up a gap. ${ }^{26}$ Therefore, the choice of Hubbard $U$ is critical to obtain a theoretical band gap close to the experimentally determined values.

Cococcioni et al. ${ }^{27}$ reported a Hubbard $\mathrm{U}$ of $3.71 \mathrm{eV}$ for $\mathrm{LiFePO}_{4}$ and $4.90 \mathrm{eV}$ for $\mathrm{FePO}_{4}$ with olivine structure calculated by a self-consistent method. Zhou et $a l .{ }^{9}$ achieved good agreement with the most accepted experimental optical gap of $3.8 \mathrm{eV}$ for $\mathrm{LiFePO}_{4}$ using GGA+U with $U=4.3 \mathrm{eV}$ (the average of $U$ values obtained in the literature ${ }^{27}$ for $\mathrm{LiFePO}_{4}$ and $\mathrm{FePO}_{4}$ ). More recently, the hybrid functional Heyd-Scuseria-Ernzerhof (HSE06) has also been used in the investigation of the electronic structure of $\mathrm{LiFePO}_{4} \cdot{ }^{28-31}$ Improved accuracy on the prediction of the band gap and the character near the Fermi level was found compared with GGA+U, along with an increase in computational cost.

In this study, $\mathrm{LiFePO}_{4}$, and its delithiated phase, $\mathrm{FePO}_{4}$, with low residual carbon were prepared using various synthesis approaches. Their band gaps were studied experimentally by UV-Vis-NIR diffusion reflectance spectroscopy and electron energy loss spectroscopy (EELS) to provide a solid basis to validate the DFT calculations. Particle surface characteristics were also examined using X-ray photoemission spectroscopy (XPS) and Raman spectroscopy. Based on results from our use of simultaneous experimental techniques, a range of functionals for DFT calculations of the band structure of olivine $\mathrm{LiFePO}_{4}$ and $\mathrm{FePO}_{4}$ have been re-evaluated, including GGA, GGA+U and HSE06. The effect of magnetic configuration and
Hubbard $\mathrm{U}$ on the estimated band gaps and band structures near the Fermi level were investigated in detail. Calculations with screened-exchange LDA (sX-LDA, CA-PZ), ${ }^{32,33}$ which is another recommended hybrid functional for more accurate band gap calculations, not used or reported previously as extensively as HSE06, were also carried out. An interpretation of open-circuit voltage of a $\mathrm{LiFePO}_{4}$ cell system has also been attempted from combined experimental inputs and electronic band structure calculation perspectives.

\section{Experimental methods}

\subsection{Sample preparation}

To investigate the band gap experimentally, $\mathrm{LiFePO}_{4}$ with low residual carbon was prepared by both hydrothermal and solution-based synthesis methods. For hydrothermal synthesis, the raw material handling was conducted under Ar atmosphere inside purged glove bag. $\mathrm{FeSO}_{4} \cdot 7 \mathrm{H}_{2} \mathrm{O}$ ( $\geq 99 \%$, Sigma-Aldrich) and $\mathrm{H}_{3} \mathrm{PO}_{4}$ (85 wt\%) were dissolved in $25 \mathrm{~mL}$ deionized water with magnetic stirring. $25 \mathrm{~mL}$ of $\mathrm{LiOH} \cdot \mathrm{H}_{2} \mathrm{O}$ (99\%, SigmaAldrich) solution was then slowly added to the above solution to bring the concentration of $\mathrm{Fe}^{2+}$ to $0.3 \mathrm{M}$ and the molar ratio Li : Fe : $\mathrm{P}=3: 1: 1$. The resulting mixture was deoxygenated using bubbling Ar for $30 \mathrm{~min}$ and then transferred into a $100 \mathrm{~mL}$ stainless steel autoclave with Teflon lining. The sealed autoclave was placed into a muffle furnace and heated up to $170{ }^{\circ} \mathrm{C}$ for $12 \mathrm{~h}$ under Ar. Subsequently, the autoclave was cooled down to room temperature before opening. The precipitates were washed six times by immersing them in deionized water and ethanol and separating the precipitates via centrifuge. For solution-based synthesis, oxalic acid dihydrate ( $\geq 99 \%$, Sigma-Aldrich) and Fe oxalate dihydrate (99\%, SigmaAldrich) were mixed in deionized water first. $30 \mathrm{wt} \% \mathrm{H}_{2} \mathrm{O}_{2}$ was slowly added into the mixture under magnetic stirring to dissolve Fe oxalate. The temperature was controlled below $65{ }^{\circ} \mathrm{C}$ during this process to avoid the formation of impurities. Stoichiometric amounts of $\mathrm{Li}_{2} \mathrm{CO}_{3}(\geq 99 \%$, Sigma-Aldrich) and $\mathrm{H}_{3} \mathrm{PO}_{4}(85 \mathrm{wt} \%)$ were added into the solution. A very small amount of polyethylene glycol (PEG) was added to obtain nano particles. The resultant clear green solution was drawn into a vessel and reacted under vacuum. The resultant precursors were ring milled and calcined at $710^{\circ} \mathrm{C}$ under Ar atmosphere to form the $\mathrm{LiFePO}_{4}$ phase. A fraction of the powders was mixed with $8 \mathrm{wt} \%$ sucrose and calcined in an Ar atmosphere for carbon coating. $\mathrm{FePO}_{4}$ was obtained by chemical delithiation of

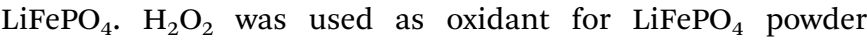
dispersed in water, as the stability of $\mathrm{LiFePO}_{4}$ in water has been reported previously. ${ }^{34}$

\subsection{Microstructural characterization}

The structure and morphology of the samples were characterized by X-ray diffraction (XRD) and scanning electron microscopy (SEM). XRD was collected on a PANalytical X'Pert pro diffractometer with $\mathrm{Co}-\mathrm{K} \alpha$ radiation, over a $2 \theta$ range between $15^{\circ}$ and $90^{\circ}$ with a $2 \theta$ step size of 0.017 . The morphology and microstructure of the samples were investigated with a JEOL 
7001 SEM. Carbon analyses were conducted using a LECO TruMac CNS analyser. The furnace temperature for the sample was $1300{ }^{\circ} \mathrm{C}$. The oxidation state of Fe was investigated with Xray photoelectron spectroscopy (XPS, Kratos AXIS Supra photoelectron spectrometer) using a focused monochromated $\mathrm{Al} \mathrm{K}_{\alpha}$ radiation $(h \nu=1486.6 \mathrm{eV})$. The spectra were calibrated using the $\mathrm{C}$ 1s peak at $285.0 \mathrm{eV}$. Particle surface characteristics were also examined with Raman spectroscopy with a Renishaw inVia Microscope equipped with a long working distance $50 \times$ objective lens and a $534 \mathrm{~nm} \mathrm{Ar}^{+}$laser light source. The power was controlled at $1 \%$ to avoid damaging the sample during measurement.

\subsection{Band gap measurements}

To validate the DFT functionals, the band gaps of $\mathrm{LiFePO}_{4}$ and $\mathrm{FePO}_{4}$ were investigated using various techniques. UV-Vis-NIR diffusion reflectance of the synthesized $\mathrm{LiFePO}_{4}$ and delithiated $\mathrm{FePO}_{4}$ were measured by a double-beam spectrophotometer Agilent Cary 5000. The wavelength range was set to 175$2800 \mathrm{~nm}$. Data were collected at a scan rate of $600 \mathrm{~nm} \mathrm{~min}{ }^{-1}$ with a data interval of $1.0 \mathrm{~nm}$, a signal bandwidth of $2.0 \mathrm{~nm}$ and signal averaging time of $0.1 \mathrm{~s}$ in the UV-Vis range. In the near IR range, the signal bandwidth was set at $4.0 \mathrm{~nm}$. During the collection, the grating and detector were changed over at $800 \mathrm{~nm}$, and the light source was changed over at $350 \mathrm{~nm}$.

Electron energy loss spectroscopy (EELS) of the $\mathrm{LiFePO}_{4}$ phase was conducted on an FEI Tecnai F20 scanning transmission electron microscope operating at $80 \mathrm{kV}$. At this operating voltage, the resolution was determined to be $0.6 \mathrm{eV}$. Lowering the acceleration voltage can minimize the beam damage and eliminate the peak caused by Cherenkov losses. ${ }^{35} \mathrm{~A}$ short exposure time of $0.5 \mathrm{~s}$ was used during the acquisition of the low energy loss spectrum. To eliminate the overlap of plasmon peaks in the low energy loss spectra, the powders were pressed into pellets and ion milled to $\sim 20 \mathrm{~nm}$ in thickness using a precision ion polishing system (Gatan, Model 691).

\section{Theoretical calculations}

All the calculations were performed within the framework of DFT using the CASTEP module in Material Studio 2017. Approaches with GGA-PBE, ${ }^{36} \mathrm{GGA}+\mathrm{U}(U=0,1,2,3,4,5,6 \mathrm{eV})$, HSE06 and SX-LDA (CA-PZ) functionals were conducted. The calculated Hubbard $\mathrm{U}$ in the literature $\left(U=3.71 \mathrm{eV}\right.$ for $\mathrm{LiFePO}_{4}$ and $U=4.90 \mathrm{eV}$ for $\left.\mathrm{FePO}_{4}\right)^{27}$ were also used for comparisons. The electronic wave functions at each $k$-point were expanded in terms of a plane-wave basis set and an energy cut-off of $900 \mathrm{eV}$ was chosen. The separation of $k$-points in GGA and GGA+U was set to $0.03 \AA^{-1}$, while that for HSE06 and sX-LDA was set to 0.07 $\AA^{-1}$ to reduce the computational cost. Norm-conserving pseudo potentials and density mixing schemes were used in the calculations when the method allowed it, otherwise, 'All Bands/ EDFT' was applied. The chosen parameters guaranteed that the total energy of the system could be evaluated accurately and the energy convergence is within $1.0 \times 10^{-6} \mathrm{eV}$ per atom. Schematics of the crystal structures of olivine $\mathrm{LiFePO}_{4}$ and $\mathrm{FePO}_{4}$ are (a)

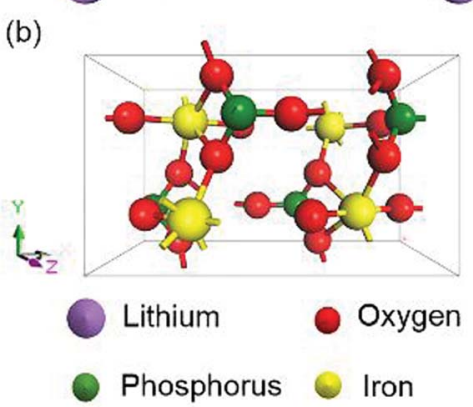

Fig. 1 Crystal structure of olivine (a) $\mathrm{LiFePO}_{4}$ and (b) $\mathrm{FePO}_{4}$ obtained from the PDF-4 database.

shown in Fig. 1. The crystal structure and lattice parameters used for calculation were obtained from the PDF-4 database. The lattice parameters for $\mathrm{LiFePO}_{4}$ at room temperature (RT) are $a=10.32525 \AA, b=6.00594 \AA$ and $c=4.69246 \AA$ with a space group of $\mathrm{Pnmb}$ (group number 62), ${ }^{37}$ while those for $\mathrm{FePO}_{4}$ are $a=9.8142 \AA, b=5.7893 \AA$ and $c=7.4820 \AA$ with a space group of Pnma (group number 62). ${ }^{38}$ Given that both $\mathrm{LiFePO}_{4}$ and $\mathrm{FePO}_{4}$ are antiferromagnetic with Neel temperatures $52 \mathrm{~K}$ and $125 \mathrm{~K}$, respectively, ${ }^{\mathbf{1 4 , 1 5}}$ ground state antiferromagnetic configurations are set up for all calculations with magnetic moment alignment based on previous literature. ${ }^{39}$ Calculations are often undertaken in the Energy setup of the calculation, where lattice parameters remain constant at the chosen RT values. Since paramagnetism is expected at RT, non-magnetic (NM) configurations have also been explored.

\section{Experimental results}

\subsection{Microstructure characterization}

The XRD pattern of the synthesized $\mathrm{LiFePO}_{4}$, together with the reference peak positions, are shown in Fig. 2a. No other peaks,
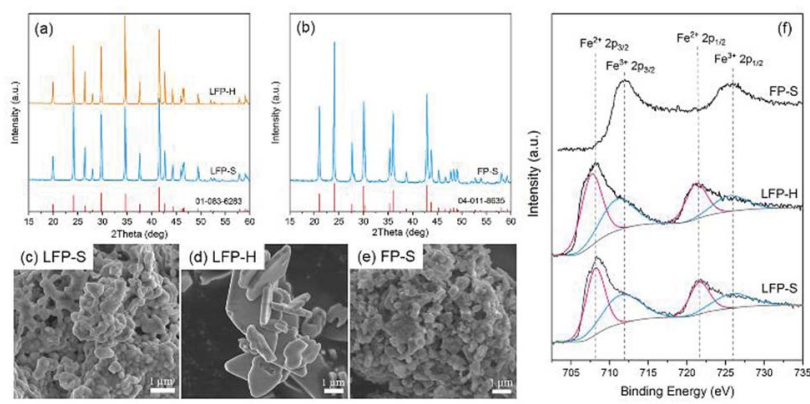

Fig. 2 (a and b) XRD patterns, (c-e) SEM micrographs and (f) XPS spectra for synthesized $\mathrm{LiFePO}_{4}$ and $\mathrm{FePO}_{4}$ samples respectively. 
except those of olivine $\mathrm{LiFePO}_{4}$, were detected for samples synthesized with both the solution-based (LFP-S) and hydrothermal (LFP-H) methods. The crystallite size calculated from XRD using the Scherrer equation was $64 \mathrm{~nm}$ for LFP-S and 74 for LFP-H. SEM micrographs are displayed in Fig. 2c and d. The LFP-S samples consisted of nano particles of $\sim 350-400 \mathrm{~nm}$ in diameter and the LFP-H samples consisted of plate-like particles of $\sim 3-6 \mu \mathrm{m}$ indicating that the particles were polycrystalline. The XRD pattern, together with the reference peak positions, and a SEM micrograph for the LFP-S samples after $48 \mathrm{~h}$ delithiation are shown in Fig. $2 \mathrm{~b}$ and e, respectively. Pure olivine $\mathrm{FePO}_{4}$, within the detection limits of XRD (about 2\%) was obtained without obvious change of the morphology of the particles and labelled as FP-S. Therefore, this delithiated sample was used to study the band structure of $\mathrm{FePO}_{4} \cdot 0.08 \%$ residual carbon was detected in both LFP-S and FP-S.

The surface oxidation states were investigated via XPS. The Fe-2p high-resolution spectra are illustrated in Fig. 2f. Around $50 \%$ of $\mathrm{Fe}$ ions were detected to be $\mathrm{Fe}^{3+}$ on the surface of both LFP-S and LFP-H particles, even though XRD indicated phase purity. In order to achieve charge neutrality, $50 \% \mathrm{Li}$ vacancies must appear on the $\mathrm{LiFePO}_{4}$ surface, which has been proven to be the most stable surface arrangement by energy minimization calculations and annular bright field (ABF) imaging in aberration-corrected scanning transmission electron microscopy (STEM) in the literature. ${ }^{40}$ On the other hand, no $\mathrm{Fe}^{2+} 2 \mathrm{p}$ peaks were detected on the surface of delithiated samples.

Raman spectroscopy has also been conducted to confirm the surface state of the samples. As shown in Fig. 3, the peak at $1068 \mathrm{~cm}^{-1}$ appears accompanied by a tiny peak at $1082 \mathrm{~cm}^{-1}$ in both $\mathrm{LiFePO}_{4}$ samples. According to the investigation of Burba et al.,$^{41}$ these peaks originate from the antisymmetric stretching mode of $\mathrm{PO}_{4}{ }^{3-}$ anion in $\mathrm{LiFePO}_{4}$ at $\sim 1072 \mathrm{~cm}^{-1}$ due to deficiency of $\mathrm{Li}$. These two peaks red-shift along with the extraction of $\mathrm{Li}$. The $1063 \mathrm{~cm}^{-1}$ and $1078 \mathrm{~cm}^{-1}$ peak are assigned to the antisymmetric stretching mode of $\mathrm{PO}_{4}{ }^{3-}$ anion in $\mathrm{FePO}_{4}$. Therefore, the existence of $\mathrm{Li}$ depletion and $\mathrm{Fe}^{3+}$ on the surface of $\mathrm{LiFePO}_{4}$ has been confirmed by both XPS and Raman spectroscopy.

\subsection{Band gap measurements}

The band gaps of $\mathrm{LiFePO}_{4}$ and $\mathrm{FePO}_{4}$ were initially investigated via UV-Vis-NIR diffusion reflectance spectroscopy. The

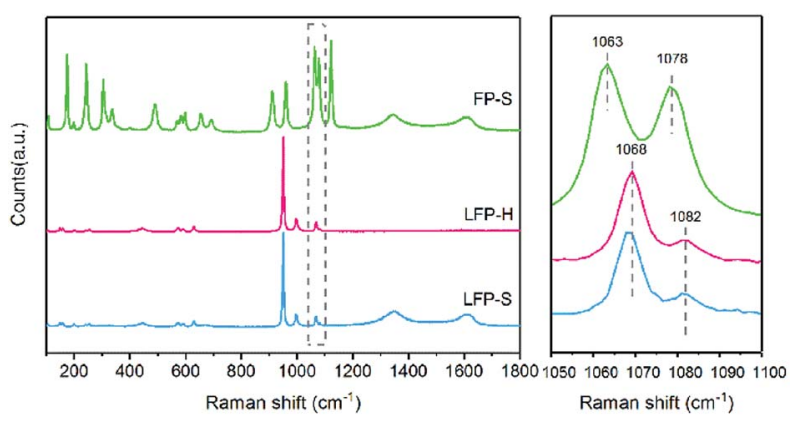

Fig. 3 Raman spectra for the synthesized samples. measured absorbance spectra and the corresponding Tauc plots ${ }^{42}$ obtained for $\mathrm{LiFePO}_{4}$ and $\mathrm{FePO}_{4}$ are shown in Fig. 4. The steps in the absorbance spectra at $\sim 1.55 \mathrm{eV}(800 \mathrm{~nm})$ were caused by the changeover of the detector. Unlike the results reported in previous literature, ${ }^{\mathbf{9 , 1 1}}$ the absorbance rose gradually in the range of 1.7-5 eV and no obvious absorbance edge in the measured energy range could be found for LFP-S. More pronounced absorption features were observed for $\mathrm{LFP}-\mathrm{H}$ samples, as a peak at $\sim 0.3 \mathrm{eV}$ and as a sharpened edge rising up at $\sim 2.8 \mathrm{eV}$, which may be caused by increased signal from (020) facets (as illustrated in the XRD spectra in Fig. 2a).

To further understand the role of residual or added carbon on the gradual absorbance increase or the position of the absorbance edge, $2.8 \mathrm{wt} \%$ carbon was coated around (labelled LFP-S/2.8C-C) or mechanically blended with (labelled LFP-S/ 2.8C-B) the LFP-S particles. An estimate of the average thickness of the carbon coating, assuming spherical $\mathrm{LiFePO}_{4}$ particles of uniform 350-400 $\mathrm{nm}$ diameter (consistent with SEM images (see Fig. 2)), results in about 2.5-2.9 $\mathrm{nm}$ thickness. Thus, as demonstrated in Fig. 4 a, a thin carbon coating $(<3 \mathrm{~nm}$ thick) largely erases the hump-shaped gradual absorption of $\mathrm{LiFePO}_{4}$ from 2.5 to $4.5 \mathrm{eV}$. Similar erasing of the absorption feature was also found in LFP-H samples, as shown in ESI Fig. S1. $\dagger$ It appears that there may be an absorption edge in $\mathrm{LiFePO}_{4}$ when the energy is higher than $6 \mathrm{eV}$ (undetectable in $\mathrm{FePO}_{4}$ ). However, the optical spectrum beyond $6.5 \mathrm{eV}$ could not be obtained due to limitations of the equipment. In contrast to the $\mathrm{LiFePO}_{4}, \mathrm{FePO}_{4}$ had a main absorption edge at $3.2 \mathrm{eV}$ with a small peak at $2.7 \mathrm{eV}$, as shown in Fig. 4b. This result is consistent with the measurements of Furutsuki et al. ${ }^{13}$ reported recently. These two characteristics became less prominent but didn't disappear or shift position with the addition of carbon, suggesting they are inherent properties of the bulk of $\mathrm{FePO}_{4}$.

Tauc plots of $(\Phi h \nu)^{n} v s$. photon energy, are widely used to interpret the UV-Vis diffusion reflectance results, where $\Phi=(1$
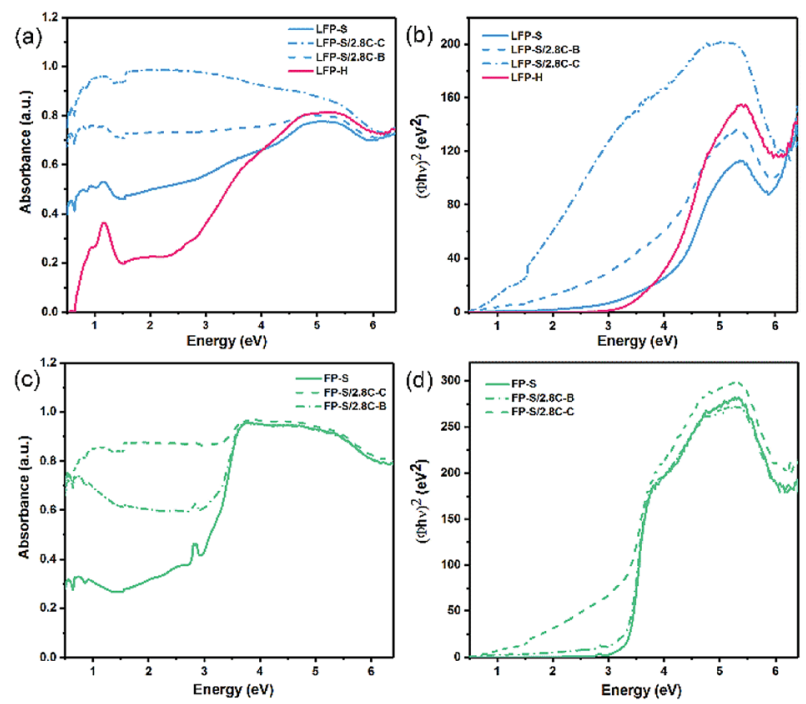

Fig. 4 UV-Vis-NIR absorbance spectra and corresponding Tauc plots for ( $a$ and b) $\mathrm{LiFePO}_{4}$ and (c and d) $\mathrm{FePO}_{4}$. 
$-R)^{2} /(2 R)$ is the Kubelka-Munk (KM) function, ${ }^{43,44} R$ is the reflectance and $n=2$ and $n=0.5$ (ref. 11) have been previously used for $\mathrm{LiFePO}_{4}$ and $\mathrm{FePO}_{4}$, respectively. In Fig. $4 \mathrm{~b}$ and d, $n=2$ is used for both $\mathrm{LiFePO}_{4}$ and $\mathrm{FePO}_{4}$, which appears to produce a sharper and more linear edge compared to $n=0.5$. The spectra of $\mathrm{FePO}_{4}$ shows clear linear edges. Extrapolation the linear part of the Tauc plot to the $x$-axis results in a band gap of $3.24 \mathrm{eV}$, which is close to the value obtained directly from the absorbance spectra. On the other hand, the Tauc plots of $\mathrm{LiFePO}_{4}$ samples show gradual absorption edges and more pronounced Urbach tails ${ }^{45}$ compared with those of $\mathrm{FePO}_{4}$ samples. Further, while the addition of carbon didn't have a significant effect on the positions of the Tauc plot edges in $\mathrm{FePO}_{4}$, the coated carbon produced a significant shift of $\sim 3 \mathrm{eV}$ toward lower energies in the Tauc plot absorption edge of LiFePO ${ }_{4}$ (see Fig. 4b), indicating that a nm-scale carbon coating has a more complex interaction with the surface of $\mathrm{LiFePO}_{4}$ particles than just producing an increased background absorption. This also opens the question as to what complex effects a nano-scale delithiated surface layer will have on the absorbance spectra of $\mathrm{LiFePO}_{4}$. It thus appears that absorption humps and tails are not part of the inherent absorption edge for the core of the $\mathrm{LiFePO}_{4}$ material, but a result of a combination of nm-scale surface layers of lithium depletion and carbon. The Tauc plots of LFP-H samples are displayed in ESI Fig. S1. $\dagger$

EELS was utilized to obtain the energy absorption beyond $6 \mathrm{eV}$ and to determine the band gap of $\mathrm{LiFePO}_{4}$. Fig. 5 illustrates the low electron energy loss spectrum (LEELS) for LFP-S. The small downward slope before $3 \mathrm{eV}$ is the tail of the zero-loss peak (ZLP). The main energy absorption starts at $6.34 \mathrm{eV}$ (as shown in Fig. 5b), indicating a band gap of $6.34 \mathrm{eV}$, which is much larger than previously reported optical results., ${ }^{\mathbf{9 1 1}}$ It is worth noticing that there is a small amount of absorption before the onset of the main absorption peak, which was consistent with the slow rise in the range of 1.55-5 eV of the UVVis-NIR absorbance spectrum of $\mathrm{LiFePO}_{4}$ in Fig. 4a. Although not measured in this paper, the LEELS spectrum for $\mathrm{FePO}_{4}$ can be found in several ref. 35 and 46-48. An energy absorption peak below $5 \mathrm{eV}$ can be found in all of the $\mathrm{FePO}_{4}$ results, although because of the existence of ZLP at $0 \mathrm{eV}$ (whose width is usually $\sim 0.2-2 \mathrm{eV}$ ), the onset of the absorption is usually merged with the tail of ZLP and hard to be determined. So EELS is not very suitable for the band gap determination when the
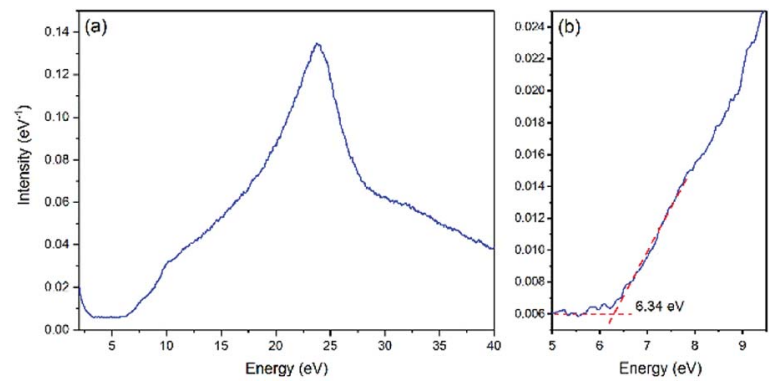

Fig. 5 (a) Low electron energy loss spectrum (LEELS) for $\mathrm{LiFePO}_{4}$. (b) Magnification of the onset of energy loss in LEELS. value is less than $5 \mathrm{eV}$. However, those absorption features in the 3-6 eV region in the EELS of $\mathrm{FePO}_{4}$ are clearly absent in the EELS of $\mathrm{LiFePO}_{4} \cdot{ }^{35,47,48}$ Thus, this is not attributable to limitations of the technique, but more likely to the fact that those peaks are not representative of the bulk, core $\mathrm{LiFePO}_{4}$ materials.

\section{Electronic structure calculations}

Since band gaps of $3.8 \mathrm{eV}$ and $1.8 \mathrm{eV}$ have been generally accepted for $\mathrm{LiFePO}_{4}$ and $\mathrm{FePO}_{4}$, respectively, many of the validations in previous literatures of the DFT functionals for the electronic band structure calculations of $\mathrm{LiFePO}_{4}$ and $\mathrm{FePO}_{4}$ have been conducted based on those values. As larger band gaps, $6.34 \mathrm{eV}$ for $\mathrm{LiFePO}_{4}$ and $3.2 \mathrm{eV}$ for $\mathrm{FePO}_{4}$, have been determined in this study, the validity of the currently used DFT functionals is questionable. Therefore, the electronic band structure calculations with various different DFT functionals, such as GGA, GGA+U and HSE06, have been revisited. The validity of sX-LDA, which has not been applied to $\mathrm{LiFePO}_{4}$ and $\mathrm{FePO}_{4}$ to our knowledge, also has been studied.

\section{1 (Semi-)local functionals}

Although the band gap underestimation for GGA has been widely reported before, the calculations on $\mathrm{LiFePO}_{4}$ and $\mathrm{FePO}_{4}$ have been revisited in this study. As both $\mathrm{LiFePO}_{4}$ and $\mathrm{FePO}_{4}$ are antiferromagnetic at $0 \mathrm{~K}$ and paramagnetic at room temperature, GGA with antiferromagnetic (AFM) and non-magnetic (NM) configurations were established to determine the effect, if any, of magnetic order on the calculated band gaps. The resulting electronic band structures are displayed in ESI Fig. S2. $\uparrow$ Similar electronic band structures with gaps of $0.50 \mathrm{eV}$ and $0.63 \mathrm{eV}$ were obtained for $\mathrm{LiFePO}_{4}$ with NM and AFM configurations, respectively, while $\mathrm{FePO}_{4}$ showed a metallic feature with NM configuration and a semiconductor feature with a band gap of $1.31 \mathrm{eV}$ with AFM configuration. Therefore, the band structure prediction of $\mathrm{FePO}_{4}$ is more affected by the inclusion of spin polarization.

Although the inclusion of spin polarization can open up the band gap to some extent, GGA is incapable of producing relatively accurate electronic structure calculations for both $\mathrm{LiFePO}_{4}$ and $\mathrm{FePO}_{4}$. The impact of the correction parameter, Hubbard U, has also been studied. Due to the necessity of spin polarization in GGA+U, AFM was set up in all of these calculations. The changing trends of estimated band gaps with respect to Hubbard $U$ are demonstrated in Fig. 6. The estimated band gap increased almost linearly along with the increase of Hubbard $\mathrm{U}$ for both $\mathrm{LiFePO}_{4}$ and $\mathrm{FePO}_{4}$ when $U$ was below $4 \mathrm{eV}$ and increased more gradually when $U$ was beyond $4 \mathrm{eV}$. The band gap of $\mathrm{LiFePO}_{4}$ showed more sensitivity to Hubbard $U$ than that of $\mathrm{FePO}_{4}$. Even though the maximum estimation of $3.96 \mathrm{eV}$ was achieved when $U=6 \mathrm{eV}$ in this work, the estimated band gap for $\mathrm{LiFePO}_{4}$ was much smaller than the experimental value of $6.34 \mathrm{eV}$. The largest gap of $2.53 \mathrm{eV}$ was obtained between the VBM and unoccupied localized Fe-3d states for $\mathrm{FePO}_{4}$, approaching the experimentally determined band gap, but still somewhat underestimated. 


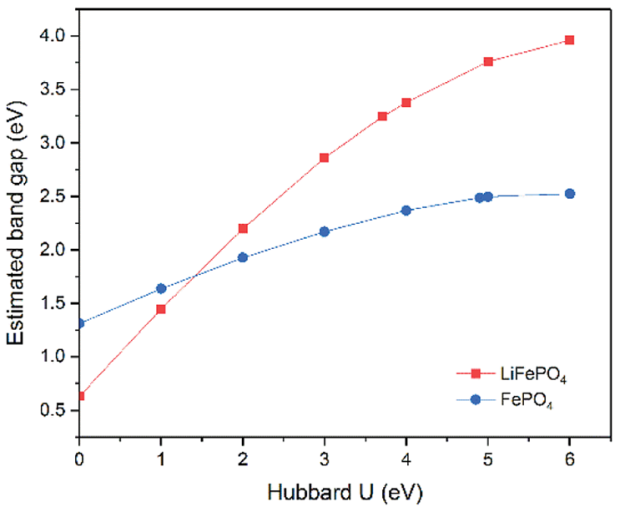

Fig. 6 Changing trends of estimated band gaps with respect to chosen Hubbard $U$ parameters. Due to the requirement of $G G A+U$, only AFM configuration was carried out.

In addition to the significant underestimation of the band gap, the orbital character of $\mathrm{LiFePO}_{4}$ near the Fermi level also changed along with the increase of Hubbard U. As shown in Fig. 7a, for olivine $\mathrm{LiFePO}_{4}$, the unoccupied $\mathrm{d}$ states were pushed upwards from the Fermi level to open up a wider band gap with the inclusion of a larger Hubbard U. In the meantime, the occupied O-2p states were also moved towards the Fermi level with the increase of $U$. When $U$ was above $4 \mathrm{eV}$, the occupied $d$ states started to align with the O-2p states. The VBM for LiFePO ${ }_{4}$ changed from occupied Fe-3d states to hybridized Fe$3 d-O-2 p$ states. O-2p states even became the majority states in the VBM when the Hubbard $\mathrm{U}$ was $6 \mathrm{eV}$, which is physically unrepresentative for $\mathrm{LiFePO}_{4}{ }^{49}$ as it will be discussed in detail later. Although the composition of the VBM and CBM didn't change, the proportion of $\mathrm{Fe}-3 \mathrm{~d}$ in the VBM also decreased with the increase of Hubbard U for $\mathrm{FePO}_{4}$ (Fig. 7b).

\subsection{Hybrid functionals}

As mentioned above, HSE06 has recently been applied to $\mathrm{LiFePO}_{4}$ and related olivine phosphates and has been proven to be superior to GGA+U. ${ }^{29-31}$ However, as another most reliable functional for electronic structure calculations and band gap determinations, ${ }^{32,50}$ sX-LDA does not appear to have been used

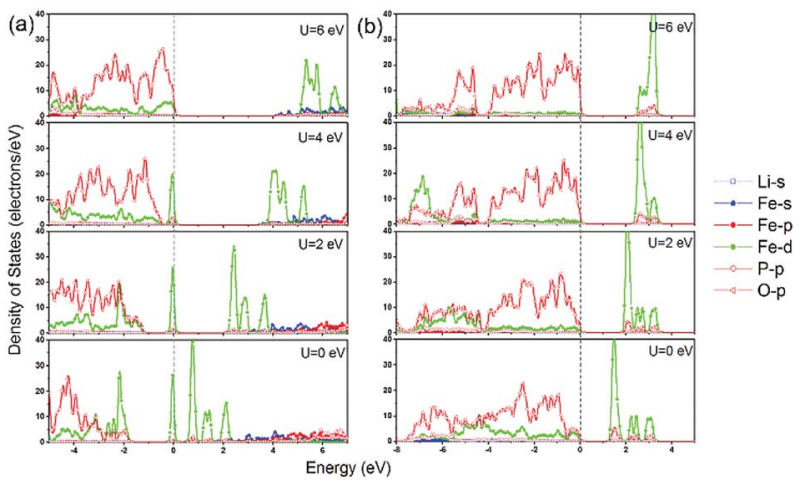

Fig. 7 Partial density of stats (PDOS) calculated in GGA and GGA+U for (a) $\mathrm{LiFePO}_{4}$ and (b) $\mathrm{FePO}_{4}$ with AFM configuration. as extensively for electronic band structure calculation for either $\mathrm{LiFePO}_{4} / \mathrm{FePO}_{4}$ or other olivine phosphates. In this study, sXLDA and HSE06 with NM and AFM configurations have been conducted for comparison. The estimated band structures and DOS with NM configurations are shown in ESI Fig. S3. $\dagger$ Similar to GGA, $\mathrm{FePO}_{4}$ showed metallic features with valence states crossing the Fermi level when excluding spin polarization within hybrid functionals, while band gaps of $3.55 \mathrm{eV}$ and $6.19 \mathrm{eV}$ between occupied and unoccupied Fe-3d states were observed for $\mathrm{LiFePO}_{4}$ within HSE06 and SX-LDA, respectively. Fig. 8 illustrates the band structures obtained by HSE06 and sXLDA with AFM configurations. The magnetic order did not have a significant effect on the electronic band structure for $\mathrm{LiFePO}_{4}$ (compare Fig. 8a, b and Fig. S3a, b $\dagger$ ). The calculated band gap of about $6.2 \mathrm{eV}$ within SX-LDA showed better agreement with the experimental value of $\mathrm{LiFePO}_{4}(6.34 \mathrm{eV})$ than that obtained by HSE06 $(3.35 \mathrm{eV})$. Hybridized Fe-3d-O-2p states were predicted for the VBM, indicating that there was partial charge transferred from oxygen to iron during delithiation, which is consistent with a recent experimental study. ${ }^{51}$ In the meantime, the inclusion of AFM order opened up a band gap of $3.34 \mathrm{eV}$ for $\mathrm{FePO}_{4}$ within SX-LDA (Fig. 8c), which also matched well with the experimental measurement. HSE06 exhibited a relatively small band gap of $1.89 \mathrm{eV}$ and a Fe-3d-dominated VBM for $\mathrm{FePO}_{4}$, which is in conflict with the experimental fact. ${ }^{35}$

For further scrutiny, the calculated DOS of HSE06, sX-LDA and GGA+U have been compared to the experimental XPS valence band spectra in Fig. 9. The Hubbard U used here is taken from prior calculated values in the literature ${ }^{27}$ Both of the hybrid functionals reproduce the valence band structures of $\mathrm{LiFePO}_{4}$ and $\mathrm{FePO}_{4}$ better than GGA+U. The relative peak positions are better reproduced using HSE06 and sX-LDA, although the spectra are slightly compressed in the range of 0-10 eV for both compounds. The spectra using GGA+U are
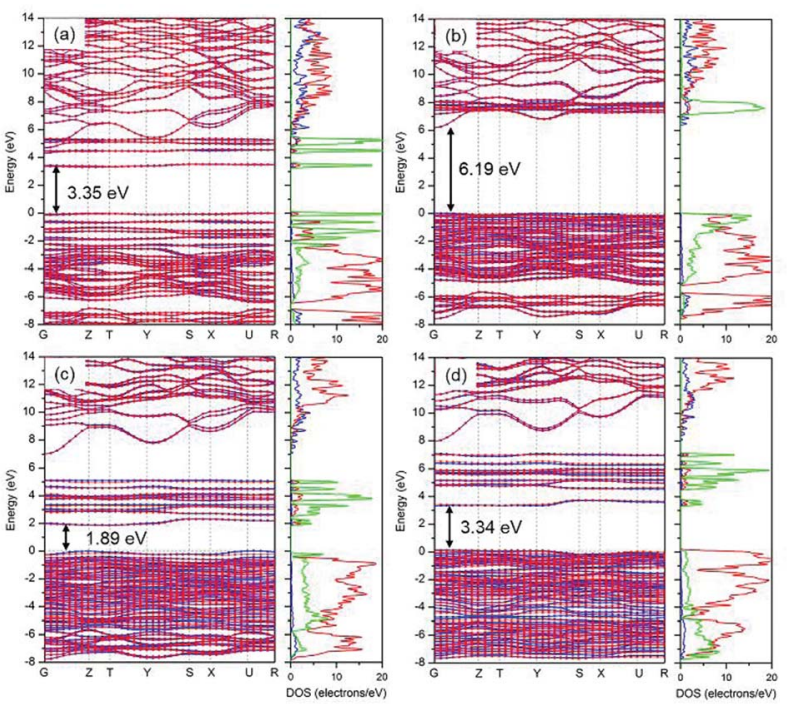

Fig. 8 Estimated band structure and DOS of ( $a$ and b) $\mathrm{LiFePO}_{4}$ and (c and b) $\mathrm{FePO}_{4}$ within HSEO6 and SX-LDA respectively. AFM configuration was applied. The blue and red lines represent the alpha and beta spin. 


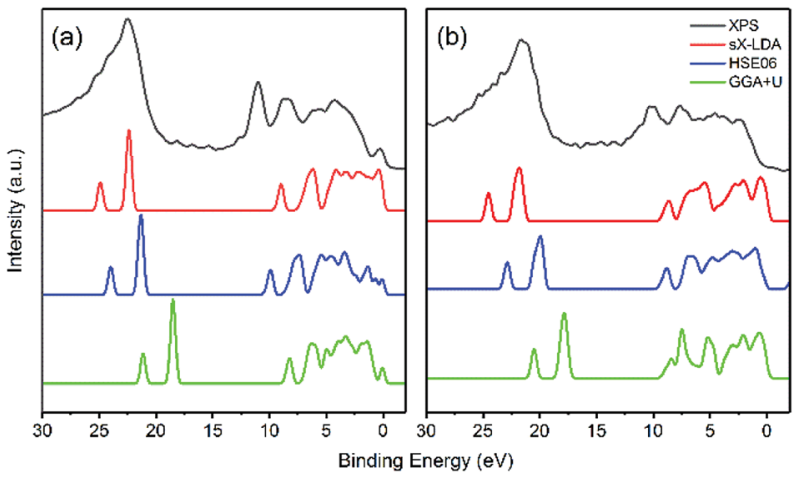

Fig. 9 Comparison of experimental valence band structure of (a) $\mathrm{LiFePO}_{4}$ and (b) $\mathrm{FePO}_{4}$ to the DOS of DFT calculations.

compressed in the whole measured energy range leading to underestimation of the binding energy.

\section{Discussions}

The following discussion first revisits the wide range of reported band gaps in Section 6.1, opening the question why such a variability exists and if there is a possible connection with some kind of undetected or uncharacterized microstructural feature. This is followed by Section 6.2, where clear evidence of delithiated surfaces in very carefully synthesized $\mathrm{LiFePO}_{4}$ samples is discussed and connected to the possibility of significant absorption edge shifts (of several eVs) by such thin layers. These results are shown to allow for a more consistent and coherent picture of combined experimental observations, both previously reported and obtained in the present study. Section 6.3 then re-assesses the DFT calculations of band gaps for $\mathrm{LiFePO}_{4}$ and $\mathrm{FePO}_{4}$ phases, in light of the extensive, combined experimental evidence, that surface delithiation is a very likely source for impurity-like in-gap states.

\subsection{Comparison of experimental band gaps}

As summarized in Table 1, the band gaps of $\mathrm{LiFePO}_{4}$ and $\mathrm{FePO}_{4}$ have been measured by several techniques. Zhou et al. ${ }^{9}$ first studied the band gap of $\mathrm{LiFePO}_{4}$ by UV-Vis-NIR diffusion reflectance spectroscopy, and a band gap of $3.8-4.0 \mathrm{eV}$ was obtained. The results by Zhou et al. had an enhanced slope around $4 \mathrm{eV}$, which looks similar to the absorption of our LFP-H samples. Unfortunately, Zhou et al.'s spectrum was truncated below $2.1 \mathrm{eV}$, not allowing full comparison with our results. We clearly see that sharpening of the $4 \mathrm{eV}$ edge is accompanied by the rise of a peak feature at about $0.6 \mathrm{eV}$ (see Fig. 4 above). Zhou et al.'s result was in good agreement with the investigation of Zaghib et al. ${ }^{11}$ which also displayed a truncated spectrum (1.8$6.2 \mathrm{eV}$ ). A band gap of $1.88 \mathrm{eV}$ for $\mathrm{FePO}_{4}$ was also determined in Zaghib et al.'s study and became the generally accepted value in following studies. However, an absorption edge at $1.88 \mathrm{eV}$ would likely result in an orange or red colour of the material, which is not consistent with the greyish colour of $\mathrm{FePO}_{4}$ powders. Recently, the band gap of $\mathrm{FePO}_{4}$ reported by Furutsuki et al. ${ }^{13}$ was $\sim 3.0 \mathrm{eV}$, which is close to the result in this study. They also claimed that the band gap of $\mathrm{LiFePO}_{4}$ was $4.8 \mathrm{eV}$, but no significant absorption was found before $5.8 \mathrm{eV}$. The features labelled as the band gap appears to be equivalent to the beginning of the Urbach tails and not the linear part of the Tauc plot. So the linear absorption, corresponding to the $5.8 \mathrm{eV}$ absorption, is more in line with our claim that the true band gap absorption edge is at $6.34 \mathrm{eV}$ (see Fig. 5).

XAS, combined with RIXS ${ }^{10}$ or XES, ${ }^{12}$ were also applied to study the electronic band structure of $\mathrm{LiFePO}_{4}$ and $\mathrm{FePO}_{4}$. Unfortunately, no specific value of band gap was clearly reported. Augustsson et al. ${ }^{12}$ claimed that the band gap could not be determined easily because of the uncertainty identifying the CBM from XAS spectra, due to the presence of a small bump between the VBM and the high intensity absorption peak. Thus, it appears that Augustsson et al. had issues with the relatively weak absorption nature of the pre-edge feature around $4 \mathrm{eV}$, and decided to label this value as the band gap, just because the absorption was detected, not further questioning a possible localized or impurity-like nature of the detected absorption. Similar pre-edge bumps have also been reported in soft X-ray absorption spectra of the $\mathrm{O}-\mathrm{K}$ edge of $\mathrm{LiFePO}_{4}$ nanoparticles by Liu $e t$ al. ${ }^{52}$ They identified them as a result from surface effects, due to the absence of this pre-edge feature in the spectra from single crystal $\mathrm{LiFePO}_{4}$. A band gap of $6.2 \mathrm{eV}$ for $\mathrm{LiFePO}_{4}$ could be obtained if we boldly assume the main absorption peak (A4 in Fig. 2 of ref. 12) in X-ray absorption-emission spectra represents the CBM, which matches well with our band gap obtained by EELS.

As mentioned above, increase of absorbance for $\mathrm{LiFePO}_{4}$ at $\sim 4 \mathrm{eV}$ was also found, but not considered as the absorption

Table 1 Comparison of band gap measurements in previous studies the present work for $\mathrm{LiFePO}_{4}$ and $\mathrm{FePO}_{4}$

\begin{tabular}{llll}
\hline & Method & Band gap & Reference \\
\hline LiFePO $_{4}$ & UV-Vis & $3.8-4.0 \mathrm{eV}$ & 9 \\
& UV-Vis & $3.84 \mathrm{eV}$ & 11 \\
& UV-Vis & $4.8 \mathrm{eV}$, but no significant absorption before $5.8 \mathrm{eV}$ & 13 \\
& XAS and RIXS & $<0.95 \mathrm{eV}$ & 10 \\
& XAS and XES & $4.0 \mathrm{or} 0.5 \mathrm{eV}$ & 12 \\
$\mathrm{FePO}_{4}$ & EELS & $6.34 \mathrm{eV}$ & This work \\
& UV-Vis & $1.88 \mathrm{eV}$ & 11 \\
& XAS and XES & $1.7 \mathrm{eV}$ & 12 \\
& UV-Vis & $\sim 3.0 \mathrm{eV}$ & 13 \\
& UV-Vis & $3.2 \mathrm{eV}$ &
\end{tabular}


edge in this work. Small amount of carbon coating or addition totally eliminate the bump in the absorption spectra of $\mathrm{LiFePO}_{4}$, or shift the linear region of the Tauc plot to lower energies by several eVs (for the coating case), making the bump look like a surface related absorption, as discussed further below.

\subsection{Surface delithiation}

As mentioned above, the surface delithiation of the $\mathrm{LiFePO}_{4}$ particles has been confirmed by XPS and Raman spectroscopy in both LFP-S and LFP-H. It is tempting to associate the surface delithiation to poor quality samples or to loss of Li from high temperature or initial off-stoichiometry in the raw material mix. However, hydrothermal samples are prepared in an excess of lithium hydroxide and at a temperature limited to $<200{ }^{\circ} \mathrm{C}$. Therefore, it appears that delithiated surfaces are a preferred equilibrium condition for good quality $\mathrm{LiFePO}_{4}$ samples. Similar surface delithiation has also been observed in other investigations. ${ }^{53,54}$

A schematic of a $\mathrm{LiFePO}_{4}$ particle surface is illustrated in Fig. 10. As detected by XPS, nearly half of Fe ions on the surface were $\mathrm{Fe}^{3+}$, suggesting a solid-solution $\mathrm{Li}_{x}\left(\mathrm{Fe}_{x}{ }^{2+} \mathrm{Fe}_{1-x}{ }^{3+}\right) \mathrm{PO}_{4}$ outer layer around the $\mathrm{LiFePO}_{4}$ core. Intervalence charge transfer has been confirmed experimentally and explained theoretically in the literature ${ }^{13}$ for $\mathrm{Li}_{0.6}\left(\mathrm{Fe}_{0.6}{ }^{2+} \mathrm{Fe}_{0.4}{ }^{3+}\right) \mathrm{PO}_{4}$ solid-solution, as the $\mathrm{Fe}^{3+}-3 \mathrm{~d}$ states fell in the gap between $\mathrm{Fe}^{2+}-3 \mathrm{~d}$ states. Similar situation can also be expected that the mixed oxidation states of Fe on the surface result in localized Fe-3d impurity states inside the band gap of bulk $\mathrm{LiFePO}_{4}$ (as shown in Fig. 10), leading to the gradual increase in optical absorption spectra in the range of 1.7-4 eV. A similar effect of a solid-solution surface on the optical absorption spectra has also been found for $\mathrm{HfO}_{2}$ with well-controlled $\mathrm{Al}$ and $\mathrm{N}$ surface diffusion. ${ }^{55}$ Nanometer-scale diffusion profiles were responsible for substantial reductions in the onset of optical absorption edge energies and for much more gradual slopes, very closely resembling the in-gap features observed in $\mathrm{LiFePO}_{4}$.

\subsection{Comparison of DFT functionals}

GGA+U, HSE06 and SX-LDA, the most practical DFT functionals for electronic band structure calculations with more reliable band gap determinations, are being compared in this section. It is worth pointing out that the dispersion of the dense VBM and

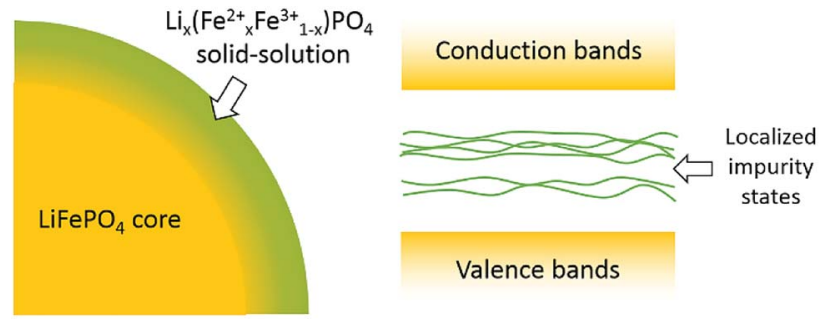

Fig. 10 Schematic of $\mathrm{LiFePO}_{4}$ particle surface and resulting electronic band structure. The existence of $\mathrm{Fe}^{3+}$ ions on the surface introduces localized impurity states in the band gap of bulk $\mathrm{LiFePO}_{4}$ which results in the gradual increase in optical absorbance spectra.
CBM regions appear similar in all the calculations and that the main differences are related to the position of the localized $\mathrm{d}$ states and to the band energy separations that define the band gaps.

Since being developed in the $1990 \mathrm{~s},{ }^{56} \mathrm{DFT}+\mathrm{U}$ has become a well-accepted functional to deal with electron correlation in transition metal and rare earth compounds. With this functional, the delocalized states are treated by conventional LDA/ GGA while the localized states are treated by inclusion of a Hubbard-like term. According to its basic features, the Hubbard model accounts for the electronic ground states through balancing two competing energies: the hopping energy and the Coulomb energy. The hopping energy includes motion of electrons with the same spin between different atoms, while the Coulomb energy represents the repulsion of electrons with opposite spin on the same site as a function of the Hubbard $\mathrm{U}$ parameter. ${ }^{57}$ Therefore, the choice of Hubbard $U$ is critical in $\mathrm{DFT}+\mathrm{U}$ calculations. However, the value of Hubbard $\mathrm{U}$ varies with atomic species, valence states of ions, lattice structures and coordination polyhedral, limiting its ab initio applications. It is thus difficult to determine a self-consistent $U$ value for a specific material. Despite this, DFT+U has been extensively used in electronic structure investigations of olivine phosphates. Although Hubbard $\mathrm{U}$ of $3.71 \mathrm{eV}$ and $4.0 \mathrm{eV}$ were calculated by Cococcioni for $\mathrm{Fe}^{2+}$ and $\mathrm{Fe}^{3+}$ in the olivine structure, ${ }^{27}$ widely different values of Hubbard $U$ have been chosen depending on the specific purpose (as summarized in Table S1 $\dagger$ ). In recent studies, $U \approx 4.5 \mathrm{eV}$ for $\mathrm{Fe}$ in $\mathrm{LiFePO}_{4}$ has become most popular because of the high accuracy based on the optical energy gap of $3.8 \mathrm{eV}$.

In this study, since the band gap of $\mathrm{LiFePO}_{4}$ was determined to be $6.34 \mathrm{eV}, \mathrm{GGA}+\mathrm{U}$ resulted in band gap underestimation even if Hubbard $\mathrm{U}$ of $6 \mathrm{eV}$ was applied. Moreover, the major atomic states character at the VBM changed with respect to the increase of Hubbard U, which also leads to problems with physico-chemical interpretation. The same changes in VBM also happens for other olivine phosphates. ESI Fig. S4 $\uparrow$ shows the PDOS of $\mathrm{LiMPO}_{4}(\mathrm{M}=\mathrm{Fe}, \mathrm{Mn}$, Co and Ni) estimated with GGA and GGA+U. The Hubbard $\mathrm{U}$ was taken from previous calculations by Cococcioni et al., ${ }^{27}$ which were obtained by a self-consistent linear response method. Except for $\mathrm{LiFePO}_{4}$, the dominant states of the VBM for all other three olivine phosphates changed to $\mathrm{O}-2 \mathrm{p}$ states after the application of Hubbard U. For $\mathrm{LiMnPO}_{4}$, even GGA provides dominant O-2p states at the VBM. In the scenario of battery application, the electrons leave the cathode with the extraction of $\mathrm{Li}^{+}$during charging, while the reverse process occurs during discharging. Accordingly, the composition of the VBM for the cathode material determines which electrons take part in the charge transfer during charging and discharging. ESI Fig. S5† illustrates schematic energy levels for a LIB system with different types of VBMs in the cathode. The partially filled transition metal 3d states (TM-3d) represent the transition metal redox couple. If the TM-3d states locate at the top of O-2p states and dominate the VBM as shown in Fig. S5a, $\uparrow$ it indicates the oxidation/reduction of the transition metal ions will occur along with the extraction/insertion of $\mathrm{Li}^{+}$during charging and 
discharging. This interpretation is consistent with the $\mathrm{LiMPO}_{4} /$ $\mathrm{MPO}_{4}$ phase transformation observed in previous studies. ${ }^{\mathbf{4} 46}$ If O-2p states take control of the VBM of the cathode, the oxidation of $\mathrm{O}^{2-}$ will lead to the release of $\mathrm{O}_{2}$ in the process of charging, which eventually leads to the collapse of the lattice structure, contradicting experimental evidence. Similar discussions have been made in previous literature. ${ }^{28,30}$ Therefore, it can be concluded that GGA+U may provide values that match some experimental determinations of band gaps, however, it does so at the expense of the DOS character, which is very important for physico-chemical interpretation. Therefore, as a whole, GGA+U may be inadequate as a DFT functional for electronic structure calculations for olivine phosphates $\mathrm{LiMPO}_{4}$ ( $\mathrm{M}=\mathrm{Fe}, \mathrm{Mn}, \mathrm{Co}$ and $\mathrm{Ni}$ ).

Improvements in band gap calculations of a wide range of semiconductors and insulators have been reported previously for HSE06 and SX-LDA. ${ }^{32,50}$ Both functionals mix a portion of Hartree-Fock (HF) exchange with (semi-)local functionals to achieve improved results. HSE functional separates the local potential into long- and short-range with the application of $25 \%$ HF exchange. ${ }^{58,59}$ For HSE, the exchange-correlation energy is determined as

$$
E_{\mathrm{XC}}^{\mathrm{HSE}}=\frac{1}{4} E_{\mathrm{X}}^{\mathrm{HF}, \mathrm{SR}}(\omega)+\left(1-\frac{1}{4}\right) E_{\mathrm{X}}^{\mathrm{PBE}, \mathrm{SR}}(\omega)+E_{\mathrm{X}}^{\mathrm{PBE}, \mathrm{LR}}+E_{\mathrm{C}}^{\mathrm{PBE}}
$$

where $E_{\mathrm{X}}^{\mathrm{HF}, \mathrm{SR}}$ is the short-range $\mathrm{HF}$ exchange energy, $E_{\mathrm{X}}^{\mathrm{PBE}, \mathrm{SR}}$ and $E_{\mathrm{X}}^{\mathrm{PBE}, \mathrm{LR}}$ are the short- and long-range $\mathrm{PBE}$ exchange energy and $E_{\mathrm{C}}^{\mathrm{PBE}, \mathrm{SR}}$ is the $\mathrm{PBE}$ correlation energy. ${ }^{59,60}$ The screening parameter $\omega$ represents the separation range, which has been determined to be $0.11 \mathrm{bohr}^{-1}$ for HSE06. ${ }^{50}$ Compared with HSE06, SX-LDA includes $100 \%$ of HF exchange, which is screened to moderate the correlation effect, into LDA. This nonlocal potential is achieved by the inclusion of a factor which decays exponentially with electron separation. Its contribution to the total energy is

$$
E_{\mathrm{nl}}^{\mathrm{sX}}=-\frac{1}{2} \sum_{i j, k q} \iint \frac{\varphi_{i k}^{*}(r) \varphi_{i k}\left(r^{\prime}\right) \exp \left(-k_{\mathrm{s}}\left|r-r^{\prime}\right|\right) \varphi_{j q}^{*}\left(r^{\prime}\right) \varphi_{j q}(r)}{\left|r-r^{\prime}\right|} \mathrm{d} r \mathrm{~d} r^{\prime}
$$

where $i$ and $j$ label electronic bands, $k$ and $q$ are $k$ points and $k_{\mathrm{s}}$ is the Thomas-Fermi screening length, ${ }^{32}$ which determines the screening range of the electron interaction and is usually evaluated from the average electron density. Thus, the screening plays a similar role to $U$ in $\mathrm{DFT}+\mathrm{U}$, by providing self-interaction correction and repulsion (or less attraction) for electrons with opposite spin at the same point. ${ }^{32,61}$ It is worth noting that sXLDA can achieve the correct asymptotic limit of the free electron gas and the SX-LDA functional is closer to the Coulombhole and screened-exchange (COHSEX) in the first-order GW method ${ }^{62}$ indicating a better performance can be expected.

Although both HSE06 and SX-LDA give relatively reasonable valence band structure compared with GGA+U, HSE06 is still not efficient enough to open up the larger band gaps of $\mathrm{LiFePO}_{4}$ and $\mathrm{FePO}_{4}$. It has been reported that the calculated band gaps are closer to experimental results when $\omega$ gets smaller in the range of $0.11-0.20 \mathrm{bohr}^{-1}$. $^{50}$ However, further decrease of $\omega$ makes the calculations difficult to converge, which leads to extremely increased computational cost. Therefore, we can conclude that SX-LDA is superior for the electronic band structure calculation of olivine $\mathrm{LiFePO}_{4}$ and $\mathrm{FePO}_{4}$. Its effectiveness on the other olivine phosphates will be further studied and reported separately.

Although hybrid functionals show overall superiority in electronic band structure calculations, their weakness is more apparent in magnetic structure identification. High spin configurations for $\mathrm{Fe}^{2+}(3 \mathrm{~d} \uparrow)^{5}(3 \mathrm{~d} \downarrow)^{1}$ and $\mathrm{Fe}^{3+}(3 \mathrm{~d} \uparrow)^{5}(3 \mathrm{~d} \downarrow)^{0}$ have been confirmed experimentally in previous literature. ${ }^{15,39,63}$ As the Fe PDOS illustrated in ESI Fig. S6, $\uparrow$ low or intermediate spin configurations $\left[(3 \mathrm{~d} \uparrow)^{3}(3 \mathrm{~d} \downarrow)^{3}\right.$ or $(3 \mathrm{~d} \uparrow)^{4}(3 \mathrm{~d} \downarrow)^{2}$ for $\mathrm{Fe}^{2+}$, and $(3 \mathrm{~d}$ $\uparrow)^{3}(3 \mathrm{~d} \downarrow)^{2}$ or $(3 \mathrm{~d} \uparrow)^{4}(3 \mathrm{~d} \downarrow)^{1}$ for $\left.\mathrm{Fe}^{3+}\right]$ have been obtained within hybrid functionals for both $\mathrm{LiFePO}_{4}$ and $\mathrm{FePO}_{4}$, even though the high spin configurations was set as input for calculations. This change in spin configuration may result from the usage of 'All Bands/EDFT' minimizer, which will ignore the atomic configurations during calculations. ${ }^{64}$ In comparison, the pre-set high spin configurations are 'stabilized' during GGA+U calculations by spin orientation and allowed transitions rules imposed as part of the construction of the methodology, which leads to a good match with experimental results.

\subsection{Open-circuit voltage}

Since the electrons transfer between cathode and anode through an external circuit along with the Li movement through the electrolyte during charging and discharging, the opencircuit voltage $\left(V_{\mathrm{OC}}\right)$ of a Li-ion battery system can be interpreted from both the perspective of the Fermi level and Gibbs free energies. ${ }^{65,66}$

It has been generally accepted that the average voltage for $\mathrm{Li}$ insertion/extraction is given after appropriate electronic band structure alignment by:

$$
V=-\frac{G\left[\mathrm{Li}_{x_{2}} \mathrm{Host}\right]-G\left[\mathrm{Li}_{x_{1}} \mathrm{Host}\right]-\left(x_{2}-x_{1}\right) G[\mathrm{Li}]}{x_{2}-x_{1}}
$$

where $G$ is the Gibbs free energy of the materials. $x_{1}=0$ and $x_{2}=1$ were typically taken in as the composition limits. Hence, the energy of $\mathrm{LiFePO}_{4}, \mathrm{FePO}_{4}$ and $\mathrm{Li}$ metal was calculated to determine the voltage for Li intercalation. As shown in Fig. 11, sX-LDA shows the highest accuracy on the estimation of $\mathrm{Li}$ intercalation voltage among the compared functionals, while GGA and HSE06 underestimate and GGA+U overestimates the $\mathrm{Li}$ intercalation voltage for $\mathrm{LiFePO}_{4}$.

From the Fermi level perspective, the $V_{\mathrm{OC}}$ can be related to the difference of Fermi levels between the cathode and anode in a cell system, after appropriate work functions alignment (with respect to the vacuum level), as shown in the equation in Fig. 12. Because the electrons flow from the cathode with lower Fermi level to the anode with higher Fermi level during charging, driven by the external charging potential, they flow back to the cathode during discharging. ${ }^{65}$ In the case of the $\mathrm{LiFePO}_{4}$ halfcell, the $V_{\mathrm{OC}}$ at the fully discharged or charged states are related to the energy difference between the Fermi level of $\mathrm{LiFePO}_{4}$ or $\mathrm{FePO}_{4}$ and that of Li metal, respectively. Fig. 12 shows a typical charging curve of a $\mathrm{LiFePO}_{4}$ half-cell and the 


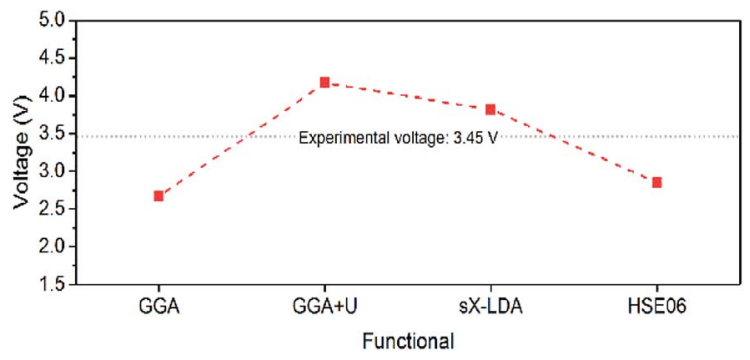

Fig. 11 Estimated Li intercalation voltage for $\mathrm{LiFePO}_{4}$ with different DFT functionals.

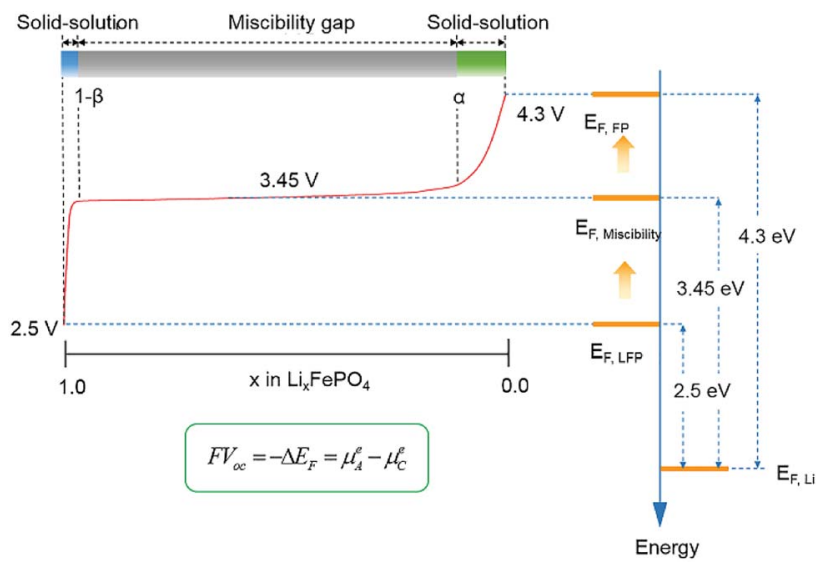

Fig. 12 Typical charging curve of a $\mathrm{LiFePO}_{4}$ half-cell with relative phase diagram and energy alignment at different charging states.

relative phase diagram. The $V_{\mathrm{OC}}$ of $\mathrm{LiFePO}_{4}$ and $\mathrm{FePO}_{4}$ were obtained experimentally in a previous investigation. ${ }^{67}$ As the Fermi level (or electrochemical potential) of Li metal does not change during charging, the $V_{\mathrm{OC}}$ differences of the fully charged and discharged state refer to the Fermi level position difference of $\mathrm{FePO}_{4}$ and $\mathrm{LiFePO}_{4}$. The Fermi energies of $\mathrm{LiFePO}_{4}$ and $\mathrm{FePO}_{4}\left(E_{\mathrm{F}, \mathrm{LFP}}\right.$ and $E_{\mathrm{F}, \mathrm{FP}}$, respectively) have been obtained with GGA theoretically, which is considered more useful for the determination of the Fermi level ${ }^{68}$ and is in reasonably good agreement with the experimental $V_{\mathrm{OC}}$ difference $(\sim 1.8 \mathrm{~V}$ in the literature ${ }^{67}$ ). The work functions of $\mathrm{LiFePO}_{4}$ and $\mathrm{FePO}_{4}$ have also been determined by ultraviolet photoelectron spectroscopy (UPS) with He I radiation (21.21 eV) experimentally. As shown in ESI Fig. S7, $\dagger$ the work functions of 5.98 and $6.82 \mathrm{eV}$ have been determined for $\mathrm{LiFePO}_{4}$ and $\mathrm{FePO}_{4}$, respectively. The result for $\mathrm{LiFePO}_{4}$ is in a good agreement with previous measurement by Kelvin Probe Force Microscopy (KPFM) ${ }^{69}$ Though the relative alignment of Fermi levels for $\mathrm{LiFePO}_{4}$ and $\mathrm{FePO}_{4}$ has been confirmed experimentally, the relatively smaller difference in experimental work functions for the two phases may be related to the morphology, atmospheric exposure during sample handling, crystal orientation, difficulties with poorly conducting materials, $e t c .^{70}$ Moreover, a work function lower that the value of the band gap appears unfeasible at first sight, however, it is not uncommon for materials with large band gaps to display negative electron affinities. ${ }^{71-73}$
A two-phase reaction has been confirmed to be responsible for the voltage plateau at $\sim 3.45 \mathrm{eV}$ for $\mathrm{LiFePO}_{4}$ in a previous study. ${ }^{74}$ From the perspective of the Fermi levels and work functions, the voltage plateau corresponds to the $\mathrm{Fe}^{2+/} \mathrm{Fe}^{3+}$ redox couple, which sits approximately at the average Fermi levels of $x \mathrm{LiFePO}_{4} /(1-x) \mathrm{FePO}_{4}\left(E_{\mathrm{F}, \text { Miscibility }}\right)$, between $E_{\mathrm{F}, \mathrm{LFP}}$ and $E_{\mathrm{F}, \mathrm{FP}}$, regardless of the proportion of the two phases. Furthermore, the plateau also provides a few hints about the relative position of $E_{\mathrm{F}, \mathrm{Li}}$ and the electronic bands of $\mathrm{LiFePO}_{4}$ and $\mathrm{FePO}_{4}$. $E_{\mathrm{F}, \mathrm{Li}}$ must be located in the forbidden gap of $x \mathrm{LiFePO}_{4} /(1-x)$ $\mathrm{FePO}_{4}$, below unoccupied d states, to achieve the flat working voltage. If the conductive bands of the cathode are located below the Fermi level of $\mathrm{Li}$, a second voltage plateau with lower electrochemical potential difference would be expected to appear in the charge-discharge curve. It is also worth noticing that the $V_{\mathrm{OC}}$ in the solid-solution regions $(0<x<\alpha$, or $1-\beta<x<$ 1) changes substantially with respect to any small change of $x$ in $\mathrm{Li}_{x} \mathrm{FePO}_{4}$. It indicates that small changes in Li concentration at the solid-solution ends have a significant impact on the position of Fermi level for $\mathrm{Li}_{x} \mathrm{FePO}_{4}$.

\section{Conclusions}

In this study, electronic band gaps of $6.34 \mathrm{eV}$ and $3.2 \mathrm{eV}$ for $\mathrm{LiFePO}_{4}$ and $\mathrm{FePO}_{4}$ were experimentally determined, respectively. The continuous energy absorption for $\mathrm{LiFePO}_{4}$ starting from $\sim 1.7 \mathrm{eV}$ was shown to be a likely consequence of the existence of $\mathrm{Fe}^{2+} / \mathrm{Fe}^{3+}$ and Li deficiency on the $\mathrm{LiFePO}_{4}$ surface. Such lithium deficiency exists at the nm or sub-nm scale and appears to be a preferred equilibrium feature, since it is present in all, including the most carefully synthesized, samples. The DFT functionals, GGA, GGA+U, HSE06 and sX-LDA, were reexamined according to the experimental band gaps. (Semi-) local functionals, GGA and GGA+U, show difficulties to achieve self-consistent prediction of the electronic structure of $\mathrm{LiFePO}_{4}$, $\mathrm{FePO}_{4}$ and other olivine phosphates $\left(\mathrm{LiMnPO}_{4}, \mathrm{LiCoPO}_{4}\right.$ and $\mathrm{LiNiPO}_{4}$ ), but succeed in stabilization of AFM configuration with high spin states. The inclusion of HF exchange within hybrid functionals can improve the valence band evaluation during electronic band structure calculation. Compared with HSE06, sX-LDA shows highest accuracy in electronic structure prediction for $\mathrm{LiFePO}_{4}$ and $\mathrm{FePO}_{4}$ with acceptable computational cost. However, both of the hybrid functionals compromise the atomic magnetic high spin order of the materials. The open-circuit voltage and charge-discharge curve of $\mathrm{LiFePO}_{4} /$ $\mathrm{FePO}_{4}$ were interpreted from a Gibbs free energy and from a Fermi levels/work functions perspective. Both of them show good agreement with experimental facts, it thus gives indirect proof of the effectiveness of SX-LDA for the theoretical calculations of $\mathrm{LiFePO}_{4}$ and $\mathrm{FePO}_{4}$. Therefore, sX-LDA can be used as an appropriate guide in future compositional and structural modifications of $\mathrm{LiFePO}_{4}$ to achieve improvements in properties such as higher power rate capability.

Besides optical diffuse reflectance measurements (which is limited to about $6 \mathrm{eV}$ with standard light sources) and EELS mentioned in the paper, large band gaps $(>6 \mathrm{eV})$ can be experimentally determined using UV ellipsometry or photo- 
conductivity measurements with a vacuum ultraviolet (VUV) monochromator ${ }^{75,76}$ Unfortunately, these suitable light sources are more specialized and not as readily available. Mirror reflectance spectra in the VUV region using synchrotron light or a deuterium lamp as an excitation source ${ }^{77}$ can also give an energy range beyond $7 \mathrm{eV}$, but single crystal samples are needed. Further clarification on the band gap of $\mathrm{LiFePO}_{4}$ and other olivine phosphates will be pursued, as soon as one of these alternative experimental techniques can be accessed or outsourced.

\section{Conflicts of interest}

There are no conflicts to declare.

\section{Acknowledgements}

Yin Zhang would like to acknowledge CSIRO for the studentship, and the involvement of CRRC and the Rail Manufacturing Cooperative Research Centre (funded jointly by participating rail organisations and the Australian Federal Government's Cooperative Research Centres Program). The experimental data reported in this paper were obtained at the Central Analytical Research Facility operated by the Institute for Future Environments, Queensland University of Technology (QUT), Brisbane, Australia. Computational resources and services used in this work were provided by the HPC and Research Support Group, QUT. The authors would like to acknowledge Mr Llew Rintoul, QUT, for the assistance of Raman spectroscopy and Prof. Ian Mackinnon, QUT, for the critical review of the manuscript.

\section{References}

1 V. Etacheri, R. Marom, R. Elazari, G. Salitra and D. Aurbach, Challenges in the development of advanced Li-ion batteries: a review, Energy Environ. Sci., 2011, 4(9), 3243-3262.

2 L. Lu, X. Han, J. Li, J. Hua and M. Ouyang, A review on the key issues for lithium-ion battery management in electric vehicles, J. Power Sources, 2013, 226, 272-288.

3 P. Canepa, G. Sai Gautam, D. C. Hannah, R. Malik, M. Liu, K. G. Gallagher, et al., Odyssey of Multivalent Cathode Materials: Open Questions and Future Challenges, Chem. Rev., 2017, 117(5), 4287-4341.

4 A. K. Padhi, K. Nanjundaswamy and J. B. Goodenough, Phospho-olivines as positive-electrode materials for rechargeable lithium batteries, J. Electrochem. Soc., 1997, 144(4), 1188-1194.

5 X.-C. Tang, L.-X. Li, Q.-L. Lai, X.-W. Song and L.-H. Jiang, Investigation on diffusion behavior of $\mathrm{Li}^{+}$in $\mathrm{LiFePO}_{4}$ by capacity intermittent titration technique (CITT), Electrochim. Acta, 2009, 54(8), 2329-2334.

6 M. Aydinol, A. Kohan, G. Ceder, K. Cho and J. Joannopoulos, $\mathrm{Ab}$ initio study of lithium intercalation in metal oxides and metal dichalcogenides, Phys. Rev. B: Condens. Matter Mater. Phys., 1997, 56(3), 1354.

7 F. Zhou, M. Cococcioni, K. Kang and G. Ceder, The Li intercalation potential of $\mathrm{LiMPO}_{4}$ and $\mathrm{LiMSiO}_{4}$ olivines with $\mathbf{M}=\mathrm{Fe}, \mathrm{Mn}, \mathrm{Co}, \mathrm{Ni}$, Electrochem. Commun., 2004, 6(11), 1144-1148.

8 Y. S. Meng and M. E. Arroyo-de Dompablo, First principles computational materials design for energy storage materials in lithium ion batteries, Energy Environ. Sci., 2009, 2(6), 589.

9 F. Zhou, K. Kang, T. Maxisch, G. Ceder and D. Morgan, The electronic structure and band gap of $\mathrm{LiFePO}_{4}$ and $\mathrm{LiMnPO}_{4}$, Solid State Commun., 2004, 132(3-4), 181-186.

10 A. Hunt, W. Y. Ching, Y. M. Chiang and A. Moewes, Electronic structures of $\mathrm{LiFePO}_{4}$ and $\mathrm{FePO}_{4}$ studied using resonant inelastic x-ray scattering, Phys. Rev. B: Condens. Matter Mater. Phys., 2006, 73(20), 205120.

11 K. Zaghib, A. Mauger, J. B. Goodenough, F. Gendron and C. Julien, Electronic, optical, and magnetic properties of $\mathrm{LiFePO}_{4}$ : small magnetic polaron effects, Chem. Mater., 2007, 19(15), 3740-3747.

12 A. Augustsson, G. V. Zhuang, S. M. Butorin, J. M. OsorioGuillen, C. L. Dong, R. Ahuja, et al., Electronic structure of phospho-olivines $\mathrm{Li}_{(\mathrm{x})} \mathrm{FePO}_{4}(\mathrm{x}=0,1)$ from soft-x-rayabsorption and -emission spectroscopies, J. Chem. Phys., 2005, 123(18), 184717.

13 S. Furutsuki, S.-C. Chung, S.-i Nishimura, Y. Kudo, K. Yamashita and A. Yamada, Electrochromism of $\mathrm{Li}_{\mathrm{x}} \mathrm{FePO}_{4}$ Induced by Intervalence Charge Transfer Transition, J. Phys. Chem. C, 2012, 116(29), 15259-15264.

14 A. Ait-Salah, J. Dodd, A. Mauger, R. Yazami, F. Gendron and C. Julien, Structural and magnetic properties of $\mathrm{LiFePO}_{4}$ and lithium extraction effects, Z. Anorg. Allg. Chem., 2006, 632(89), 1598-1605.

15 C. M. Julien, A. Ait-Salah, A. Mauger and F. Gendron, Magnetic properties of lithium intercalation compounds, Ionics, 2006, 12(1), 21-32.

16 M. Milović, D. Jugović, N. Cvjetićanin, D. Uskoković, A. S. Milošević, Z. S. Popović, et al., Crystal structure analysis and first principle investigation of $\mathrm{F}$ doping in $\mathrm{LiFePO}_{4}$, J. Power Sources, 2013, 241, 70-79.

17 Y.-N. Xu, S.-Y. Chung, J. T. Bloking, Y.-M. Chiang and W. Y. Ching, Electronic Structure and Electrical Conductivity of Undoped $\mathrm{LiFePO}_{4}$, Electrochem. Solid-State Lett., 2004, 7(6), A131.

18 J. Jiang, C. Ouyang, H. Li, Z. Wang, X. Huang and L. Chen, First-principles study on electronic structure of $\mathrm{LiFePO}_{4}$, Solid State Commun., 2007, 143(3), 144-148.

19 C. Ban, W.-J. Yin, H. Tang, S.-H. Wei, Y. Yan and A. C. Dillon, A Novel Codoping Approach for Enhancing the Performance of $\mathrm{LiFePO}_{4}$ Cathodes, Adv. Energy Mater., 2012, 2(8), 10281032.

20 T.-F. Yi, Z.-K. Fang, Y. Xie, Y.-R. Zhu and C. Dai, Band structure analysis on olivine $\mathrm{LiMPO}_{4}$ and delithiated $\mathrm{MPO}_{4}$ $(\mathrm{M}=\mathrm{Fe}, \mathrm{Mn})$ cathode materials, J. Alloys Compd., 2014, 617, 716-721.

21 Y.-N. Xu, W. Y. Ching and Y.-M. Chiang, Comparative studies of the electronic structure of $\mathrm{LiFePO}_{4}, \mathrm{FePO}_{4}, \mathrm{Li}_{3} \mathrm{PO}_{4}$, $\mathrm{LiMnPO}_{4}, \mathrm{LiCoPO}_{4}$, and $\mathrm{LiNiPO}_{4}$, J. Appl. Phys., 2004, 95(11), 6583-6585. 
22 F. Giustino, Materials modelling using density functional theory: properties and predictions, Oxford University Press, 2014.

23 A. Yamada and S.-C. Chung, Crystal Chemistry of the Olivine-Type $\mathrm{Li}\left(\mathrm{Mn}_{\mathrm{y}} \mathrm{Fe}_{1-\mathrm{y}}\right) \mathrm{PO}_{4}$ and $\left(\mathrm{Mn}_{\mathrm{y}} \mathrm{Fe}_{1-\mathrm{y}}\right) \mathrm{PO}_{4}$ as Possible $4 \mathrm{~V}$ Cathode Materials for Lithium Batteries, $J$. Electrochem. Soc., 2001, 148(8), A960.

24 S. Shi, L. Liu, C. Ouyang, D.-s Wang, Z. Wang, L. Chen, et al., Enhancement of electronic conductivity of $\mathrm{LiFePO}_{4}$ by $\mathrm{Cr}$ doping and its identification by first-principles calculations, Phys. Rev. B: Condens. Matter Mater. Phys, 2003, 68(19), 195108.

25 P. Tang and N. A. W. Holzwarth, Electronic structure of $\mathrm{FePO}_{4}, \mathrm{LiFePO}_{4}$, and related materials, Phys. Rev. B: Condens. Matter Mater. Phys., 2003, 68(16), 165107.

26 V. I. Anisimov, J. Zaanen and O. K. Andersen, Band theory and Mott insulators: Hubbard U instead of Stoner I, Phys. Rev. B: Condens. Matter Mater. Phys., 1991, 44(3), 943.

27 F. Zhou, M. Cococcioni, C. A. Marianetti, D. Morgan and G. Ceder, First-principles prediction of redox potentials in transition-metal compounds with LDA+U, Phys. Rev. B: Condens. Matter Mater. Phys., 2004, 70(23), 235121.

28 V. L. Chevrier, S. P. Ong, R. Armiento, M. K. Y. Chan and G. Ceder, Hybrid density functional calculations of redox potentials and formation energies of transition metal compounds, Phys. Rev. B: Condens. Matter Mater. Phys., 2010, 82(7), 075122.

29 S. P. Ong, V. L. Chevrier and G. Ceder, Comparison of small polaron migration and phase separation in olivine $\mathrm{LiMnPO}_{4}$ and $\mathrm{LiFePO}_{4}$ using hybrid density functional theory, Phys. Rev. B: Condens. Matter Mater. Phys., 2011, 83(7), 075112.

30 M. D. Johannes, K. Hoang, J. L. Allen and K. Gaskell, Hole polaron formation and migration in olivine phosphate materials, Phys. Rev. B: Condens. Matter Mater. Phys., 2012, 85(11), 115106.

31 M. Nakayama, S. Yamada, R. Jalem and T. Kasuga, Density functional studies of olivine-type $\mathrm{LiFePO}_{4}$ and $\mathrm{NaFePO}_{4}$ as positive electrode materials for rechargeable lithium and sodium ion batteries, Solid State Ionics, 2016, 286, 40-44.

32 S. J. Clark and J. Robertson, Screened exchange density functional applied to solids, Phys. Rev. B: Condens. Matter Mater. Phys., 2010, 82(8), 085208.

33 R. Gillen and J. Robertson, Accurate screened exchange band structures for the transition metal monoxides $\mathrm{MnO}, \mathrm{FeO}$, CoO and NiO, J. Phys.: Condens. Matter, 2013, 25(16), 165502.

34 K. Zaghib, M. Dontigny, P. Charest, J. Labrecque, A. Guerfi, M. Kopec, et al., Aging of $\mathrm{LiFePO}_{4}$ upon exposure to $\mathrm{H}_{2} \mathrm{O}$, J. Power Sources, 2008, 185(2), 698-710.

35 M. K. Kinyanjui, P. Axmann, M. Wohlfahrt-Mehrens, P. Moreau, F. Boucher and U. Kaiser, Origin of valence and core excitations in $\operatorname{LiFePO}(4)$ and $\operatorname{FePO}(4), J$. Phys.: Condens. Matter, 2010, 22(27), 275501.

36 J. P. Perdew, K. Burke and M. Ernzerhof, Generalized gradient approximation made simple, Phys. Rev. Lett., 1996, 77(18), 3865.

37 Y. Mishima, T. Hojo, T. Nishio, H. Sadamura, N. Oyama, C. Moriyoshi, et al., MEM Charge Density Study of Olivine
$\mathrm{LiMPO}_{4}$ and $\mathrm{MPO}_{4}(\mathrm{M}=\mathrm{Mn}, \mathrm{Fe})$ as Cathode Materials for Lithium-Ion Batteries, J. Phys. Chem. C, 2013, 117(6), 26082615.

38 A. S. Andersson, B. Kalska, L. Häggström and J. O. Thomas, Lithium extraction/insertion in $\mathrm{LiFePO}_{4}$ : an X-ray diffraction and Mössbauer spectroscopy study, Solid State Ionics, 2000, 130(1), 41-52.

39 G. Rousse, J. Rodriguez-Carvajal, S. Patoux and C. Masquelier, Magnetic structures of the triphylite $\mathrm{LiFePO}_{4}$ and of its delithiated form $\mathrm{FePO}_{4}$, Chem. Mater., 2003, 15(21), 4082-4090.

40 S. Kobayashi, C. A. Fisher, T. Kato, Y. Ukyo, T. Hirayama and Y. Ikuhara, Atomic-Scale Observations of (010) $\mathrm{LiFePO}_{4}$ Surfaces Before and After Chemical Delithiation, Nano Lett., 2016, 16(9), 5409-5414.

41 C. M. Burba and R. Frech, Raman and FTIR Spectroscopic Study of $\mathrm{Li}_{\mathrm{x}} \mathrm{FePO}_{4}(0 \leq \mathrm{x} \leq 1)$, J. Electrochem. Soc., 2004, 151(7), A1032-A1038.

$42 \mathrm{~J}$. Tauc, Optical properties and electronic structure of amorphous Ge and Si, Mater. Res. Bull., 1968, 3(1), 37-46.

43 P. Kubelka and F. Munk, An article on optics of paint layers, Z. Tech. Phys., 1931, 12, 593-601.

44 P. Kubelka, New contributions to the optics of intensely light-scattering materials. Part I, J. Opt. Soc. Am., 1948, 38(5), 448-457.

45 M. Schreiber and Y. Toyozawa, Numerical experiments on the absorption lineshape of the exciton under lattice vibrations. III. The Urbach rule, J. Phys. Soc. Jpn., 1982, 51(5), 1544-1550.

46 M. Lachal, R. Bouchet, A. Boulineau, S. Surblé, C. Rossignol, F. Alloin, et al., Remarkable impact of grains boundaries on the chemical delithiation kinetics of $\mathrm{LiFePO}_{4}$, Solid State Ionics, 2017, 300, 187-194.

47 P. Moreau, V. Mauchamp, F. Pailloux and F. Boucher, Fast determination of phases in $\mathrm{Li}_{\mathrm{x}} \mathrm{FePO}_{4}$ using low losses in electron energy-loss spectroscopy, Appl. Phys. Lett., 2009, 94(12), 123111.

48 W. Sigle, R. Amin, K. Weichert, P. A. van Aken and J. Maier, Delithiation Study of $\mathrm{LiFePO}_{4}$ Crystals Using Electron Energy-Loss Spectroscopy, Electrochem. Solid-State Lett., 2009, 12(8), A151.

49 C. Julien, A. Mauger, K. Zaghib and H. Groult, Comparative Issues of Cathode Materials for Li-Ion Batteries, Inorganics, 2014, 2(1), 132.

50 A. V. Krukau, O. A. Vydrov, A. F. Izmaylov and G. E. Scuseria, Influence of the exchange screening parameter on the performance of screened hybrid functionals, J. Chem. Phys., 2006, 125(22), 224106.

51 S. Miao, M. Kocher, P. Rez, B. Fultz, R. Yazami and C. C. Ahn, Local Electronic Structure of Olivine Phases of $\mathrm{Li}_{\mathrm{x}} \mathrm{FePO}_{4}, \mathrm{~J}$. Phys. Chem. A, 2007, 111(20), 4242-4247.

52 X. Liu, Y. J. Wang, B. Barbiellini, H. Hafiz, S. Basak, J. Liu, et al., Why $\mathrm{LiFePO}_{4}$ is a safe battery electrode: Coulomb repulsion induced electron-state reshuffling upon lithiation, Phys. Chem. Chem. Phys., 2015, 17(39), 2636926377. 
53 L. Castro, R. Dedryvère, J.-B. Ledeuil, J. Bréger, C. Tessier and D. Gonbeau, Aging mechanisms of $\mathrm{LiFePO}_{4} / /$ graphite cells studied by XPS: redox reaction and electrode/electrolyte interfaces, J. Electrochem. Soc., 2012, 159(4), A357-A363.

54 H. Shang, W. Chu, J. Cheng, F. Pan, D. Cheng, D. Xia, et al., Surface phase composition of nanosized $\mathrm{LiFePO}_{4}$ and their enhanced electrochemical properties, J. Mater. Chem. A, 2013, 1(22), 6635-6641.

55 P. S. Lysaght, J. C. Woicik, M. A. Sahiner, J. Price, C. Weiland and P. D. Kirsch, Spectroscopic analysis of $\mathrm{Al}$ and $\mathrm{N}$ diffusion in $\mathrm{HfO}_{2}$, J. Appl. Phys., 2012, 112(6), 064118.

56 A. I. Liechtenstein, V. I. Anisimov and J. Zaanen, Densityfunctional theory and strong interactions: Orbital ordering in Mott-Hubbard insulators, Phys. Rev. B: Condens. Matter Mater. Phys., 1995, 52(8), R5467-R5470.

57 J. Stöhr and H. C. Siegmann, Magnetism: from fundamentals to nanoscale dynamics, Springer Science \& Business Media, 2007.

58 J. Heyd, G. E. Scuseria and M. Ernzerhof, Hybrid functionals based on a screened Coulomb potential,J. Chem. Phys., 2003, 118(18), 8207-8215.

59 J. Heyd and G. E. Scuseria, Efficient hybrid density functional calculations in solids: Assessment of the HeydScuseria-Ernzerhof screened Coulomb hybrid functional, $J$. Chem. Phys., 2004, 121(3), 1187-1192.

60 J. P. Perdew, M. Ernzerhof and K. Burke, Rationale for mixing exact exchange with density functional approximations, J. Chem. Phys., 1996, 105(22), 9982-9985.

61 B. Lee, L.-W. Wang, C. D. Spataru and S. G. Louie, Nonlocal exchange correlation in screened-exchange density functional methods, Phys. Rev. B: Condens. Matter Mater. Phys., 2007, 76(24), 245114.

$62 \mathrm{H}$. Li, R. Gillen and J. Robertson, The screening effects of the screened exchange hybrid functional in surface systems: A case study on the CO/Pt(111) problem, AIP Adv., 2016, 6(6), 065309.

63 G. Liang, K. Park, J. Li, R. E. Benson, D. Vaknin, J. T. Markert, et al., Anisotropy in magnetic properties and electronic structure of single-crystal $\mathrm{LiFePO}_{4}$, Phys. Rev. B: Condens. Matter Mater. Phys., 2008, 77(6), 064414.

64 CASTEP Guide Materials Studio 8.0, http:// www.tcm.phy.cam.ac.uk/castep/documentation/WebHelp/ content/pdfs/castep.htm.

65 J. Gao, S.-Q. Shi and H. Li, Brief overview of electrochemical potential in lithium ion batteries, Chin. Phys. B, 2016, 25(1), 018210.
66 A. Urban, D.-H. Seo and G. Ceder, Computational understanding of Li-ion batteries, npj Comput. Mater., 2016, 2(1), 16002.

67 G. Kobayashi, S.-i Nishimura, M.-S. Park, R. Kanno, M. Yashima, T. Ida, et al., Isolation of Solid Solution Phases in Size-Controlled $\mathrm{Li}_{\mathrm{x}} \mathrm{FePO}_{4}$ at Room Temperature, Adv. Funct. Mater., 2009, 19(3), 395-403.

68 M. M. Kalantarian, S. Asgari and P. Mustarelli, A theoretical approach to evaluate the rate capability of Li-ion battery cathode materials, J. Mater. Chem. A, 2014, 2(1), 107-115.

69 W. Yang, Z. Wang, L. Chen, Y. Chen, L. Zhang, Y. Lin, et al., Suppression of degradation for lithium iron phosphate cylindrical batteries by nano silicon surface modification, RSC Adv., 2017, 7(53), 33680-33687.

70 A. Kahn, Fermi level, work function and vacuum level, Mater. Horiz., 2016, 3(1), 7-10.

71 D. T. Pierce, F. Meier and P. Zürcher, Negative electron affinity GaAs: A new source of spin-polarized electrons, Appl. Phys. Lett., 1975, 26(12), 670-672.

72 J. van der Weide, Z. Zhang, P. K. Baumann, M. G. Wensell, J. Bernholc and R. J. Nemanich, Negative-electron-affinity effects on the diamond (100) surface, Phys. Rev. B: Condens. Matter Mater. Phys., 1994, 50(8), 5803-5806.

73 M. Powers, M. Benjamin, L. Porter, R. Nemanich, R. Davis, J. Cuomo, et al., Observation of a negative electron affinity for boron nitride, Appl. Phys. Lett., 1995, 67(26), 3912-3914.

74 M. Takahashi, S. Tobishima, K. Takei and Y. Sakurai, Characterization of $\mathrm{LiFePO}_{4}$ as the cathode material for rechargeable lithium batteries, J. Power Sources, 2001, 97, 508-511.

75 S.-G. Lim, S. Kriventsov, T. N. Jackson, J. H. Haeni, D. G. Schlom, A. M. Balbashov, et al., Dielectric functions and optical bandgaps of high-K dielectrics for metal-oxidesemiconductor field-effect transistors by far ultraviolet spectroscopic ellipsometry, J. Appl. Phys., 2002, 91(7), 45004505.

76 J. Selj, T. Mongstad, B. Hauback and S. Z. Karazhanov, The dielectric functions and optical band gaps of thin films of amorphous and cubic crystalline $\mathrm{Mg}_{\sim 2} \mathrm{NiH}_{\sim 4}$, Thin Solid Films, 2012, 520(22), 6786-6792.

77 D. Spassky, N. Kozlova, V. Nagirnyi, A. Savon, Y. A. Hizhnyi and S. Nedilko, Excitation energy transfer to luminescence centers in $\mathrm{M}^{\mathrm{II}} \mathrm{MoO}_{4}\left(\mathrm{M}^{\mathrm{II}}=\mathrm{Ca}, \mathrm{Sr}, \mathrm{Zn}, \mathrm{Pb}\right)$ and $\mathrm{Li}_{2} \mathrm{MoO}_{4}, J$. Lumin., 2017, 186, 229-237. 